\title{
ASSISTÊNCIA DE ENFERMAGEM SEGURA E QUALIFICADA: AVALIAÇÃO DO RISCO CIRÚRGICO NO CUIDADO PERIOPERATÓRIO AO IDOSO
}

\author{
Melissa Orlandi Honório Locks ${ }^{1}$, Darla Lusia Ropelato Fernandez ${ }^{2}$, Lúcia Nazareth Amante ${ }^{1}$, Karina Silveira \\ de Almeida Hammerschmidt ${ }^{1}$, Luciara Fabiane Sebold ${ }^{1}$, Juliana Balbinot Reis Girondi ${ }^{1}$
}

RESUMO: Objetivou-se discutir o uso de escalas para avaliar o risco cirúrgico de idosos no período perioperatório. Trata-se de comentário crítico fundamentado nos princípios da segurança do paciente e no cuidado à pessoa idosa submetida a procedimento cirúrgico uma vez que o enfermeiro realiza avaliação global, identificando os fatores de risco e prevenindo complicações cirúrgicas. A busca na literatura científica foi realizada entre os meses de julho e agosto de 2015. Os resultados evidenciam que escalas preditoras são tecnologias de cuidado que devem ser utilizadas pelos enfermeiros no cuidado ao idoso no período perioperatório com foco na cirurgia segura. Conclui-se que as escalas para avaliação de risco cirúrgico apresentadas podem ser incorporadas à prática cotidiana de cuidado dos enfermeiros, mediante o estabelecimento de plano de cuidados individualizado. A Enfermagem precisa instrumentalizar-se nesse processo e contribuir para a produção do conhecimento nesta perspectiva, a fim de fundamentar e implementar intervenções baseadas em evidências científicas.

DESCRITORES: Enfermagem; Segurança do paciente; Idoso; Período perioperatório.

\section{SAFE AND EFFECTIVE NURSING CARE: ASSESSMENT OF SURGICAL RISKS IN PERIOPERATIVE CARE OF ELDERLY PATIENTS}

\begin{abstract}
The present study aimed to examine the use of scales for assessment of surgical risks for elderly patients in the perioperative period. It involves a critical analysis of the principles of patient safety and care provided to elderly individuals undergoing surgical procedures, since nurses perform a comprehensive assessment in order to identify risk factors and prevent surgical complications. Search of scientific literature was performed in July and August 2015. The results obtained indicated that scales for predicting risks are patient care technologies that should be used by nurses in the perioperative period, in order to ensure a safe surgery. It is concluded that these scales can be used in the daily practice of nurses, through the establishment of an individualized care plan. Nursing should use this approach and contribute to the production of knowledge under this perspective, in order to implement interventions based on scientific evidence.
\end{abstract}

DESCRIPTORS: Nursing; Patient safety; Elderly; Perioperative period.

\section{ASISTENCIA DE ENFERMERÍA SEGURA Y CUALIFICADA: EVALUACIÓN DEL RIESGO QUIRÚRGICO EN EL CUIDADO PERIOPERATORIO AL ANCIANO}

RESUMEN: Fue objetivo del estudio discutir el uso de escalas para evaluar el riesgo quirúrgico de ancianos en el periodo perioperatorio. Es un comentario crítico fundamentado en los principios de la seguridad del paciente y en el cuidado a la persona mayor sometida a procedimiento quirúrgico, considerando que el enfermero realiza evaluación global, identificando los factores de riesgo y previniendo complicaciones quirúrgicas. La búsqueda en la literatura científica fue realizada entre los meses de julio y agosto de 2015. Los resultados muestran que escalas predictoras son tecnologías de cuidado que deben ser utilizadas por los enfermeros en el cuidado al anciano en el periodo perioperatorio con foco en la cirugía segura. Se concluye que las escalas para evaluación de riesgo quirúrgico presentadas pueden ser incorporadas a la práctica cotidiana de cuidado de los enfermeros, por medio del establecimiento de planes de cuidados individualizados. La Enfermería necesita de instrumentalización en ese proceso, así como contribuir para la producción del conocimiento en esta perspectiva, para fundamentar y implementar intervenciones basadas en evidencias científicas.

DESCRIPTORES: Enfermería; Seguridad del paciente; Anciano; Periodo perioperatorio.

${ }^{1}$ Enfermeira. Doutora em Enfermagem. Docente de Enfermagem da Universidade Federal de Santa Catarina. Florianópolis, SC, Brasil.

${ }^{2}$ Enfermeira. Doutoranda do Programa de Pós-Graduação em Enfermagem da Universidade Federal de Santa Catarina. Docente de Enfermagem da Universidade Federal de Santa Catarina. Florianópolis, SC, Brasil.

Autor Correspondente:

Juliana Balbinot Reis Girondi

Universidade Federal de Santa Catarina

R. Delminda Silveira, 363 - 88025-500 - Florianópolis, SC, Brasil

E-mail: juliana.balbinot@ufsc.br
Recebido: $17 / 02 / 2016$

Finalizado: 14/09/2016 


\section{INTRODUÇÃO}

Intervenções cirúrgicas em idosos são cada vez mais frequentes pelo envelhecimento populacional e aumento das morbidades de resolução cirúrgica. Consoante, agudizações e complicações crônicas associadas às alterações próprias do envelhecimento são fatores indispensáveis a serem considerados no período perioperatório ${ }^{(1-2)}$. A complexidade do ambiente cirúrgico e os riscos inerentes à cirurgia são domínios que refletem na necessidade de organização adequada para garantir segurança no cuidado ao paciente ${ }^{(3)}$.

Nesta perspectiva, o enfermeiro possui importante atuação, seus cuidados poderão oportunizar um período perioperatório com menos intercorrências e maior sobrevida. Promove-se assim o cuidado resolutivo, com qualidade, preservando a segurança do paciente, principalmente evocando da enfermagem capacidade científica e comprometimento com ações sistêmicas de avaliação, na tentativa de viabilizar a redução de desfechos indesejados e prevenir complicações ${ }^{(4)}$.

A incidência de complicações perioperatórias está diretamente relacionada à idade, presença de comorbidades e urgência cirúrgica. Logo, o idoso é mais suscetível e vulnerável à morbimortalidade, requerendo maior atenção por parte dos profissionais de saúde ${ }^{(1,5-6)}$. Ressalta-se que idosos constituem grupo heterogêneo, especialmente na atualidade, quando o conceito de idade funcional tem estreita relação com o envelhecimento biológico, o que é bastante peculiar em cada ser humano ${ }^{(7)}$.

Neste sentido, uma das estratégias de cuidado a ser empregada pela equipe de saúde que atende o idoso no período perioperatório é a utilização de escalas para avaliação do risco cirúrgico. Estas permitem calcular a probabilidade do paciente apresentar complicações no pós-operatório, já que esse alto risco exige uma série de cuidados para que se possa prepará-los adequadamente para os procedimentos $^{(1)}$.

Algumas escalas têm sido estudadas e utilizadas para esta avaliação com implicações prognósticas em relação ao ato operatório( ${ }^{(1)}$. Entretanto, estas não apresentam foco específico para o idoso, sendo necessária a utilização concomitante de diversos instrumentos para obter-se um prognóstico completo da situação.

Nesta perspectiva, este estudo tem por objetivo discutir o uso de escalas para avaliar o risco cirúrgico de idosos no período perioperatório, vislumbrando avaliação integral e assistência de enfermagem segura e de qualidade.

\section{MÉTODO}

Trata-se de comentário crítico, compreendido como recurso reflexivo e argumentativo(8). Para tanto, buscou-se na literatura científica, entre os meses de julho a agosto de 2015, estudos sobre escalas de avaliação dos riscos cirúrgicos. Assim, foram pesquisadas as base de dados da Literatura Latino-americana e do Caribe em Ciências da Saúde (LILACS) e do Sistema Online de Busca e Análise de Literatura Médica (MEDLINE) utilizando os descritores: avaliação de risco e assistência no período perioperatório. Após o processo de busca, realizou-se análise individualizada do estudo, com destaque para o tipo de risco que a escala avalia e de que forma é realizada esta ponderação. Como resultado, elaborou-se um quadro com os instrumentos encontrados, seguido de discussão teórico científica, focada na utilização destas para os idosos em condição perioperatória.

\section{RESULTADOS}

Existem várias escalas de risco cirúrgico que possuem implicações prognósticas em relação ao ato operatório, contudo, nenhuma delas contemplam isoladamente a totalidade das demandas de riscos e cuidados apresentadas pelos idosos ${ }^{(1)}$. O Quadro1 apresenta as principais escalas identificadas neste estudo.

Para avaliar globalmente o idoso em condição cirúrgica, o enfermeiro deverá ter a capacidade 
Quadro 1 - Escalas para avaliação de riscos cirúrgicos. Florianópolis, SC, Brasil, 2016 (continua)

\begin{tabular}{|c|c|c|}
\hline Risco Avaliado & Código do Instrumento & Fonte \\
\hline \multirow[t]{2}{*}{$\begin{array}{l}\text { Risco Cirúrgico } \\
\text { Global }\end{array}$} & E1 - Índice de Goldman & $\begin{array}{l}\text { Goldman L, Caldera DL, Nussbaum SR, Southwick FS, Krogstad D, } \\
\text { Murray B,et al. Multifactorial index of cardiac risk in noncardiac } \\
\text { surgical procedures. N Engl J Med. } 1977 ; 297(16): 845-50^{(9)} \text {. }\end{array}$ \\
\hline & $\begin{array}{l}\text { E2 - American Society of } \\
\text { Anesthesiologists ASA }\end{array}$ & $\begin{array}{l}\text { Owens William D. American Society of Anesthesiologists. } \\
\text { Physical Status Classification System Is Not a Risk Classification } \\
\text { System. Anesthesiolog. } 2001 ; 94(2): 378^{(10)} \text {. }\end{array}$ \\
\hline \multirow[t]{2}{*}{$\begin{array}{l}\text { Risco Cirúrgico } \\
\text { Cardíaco }\end{array}$} & E3 - Índice de Detsky & $\begin{array}{l}\text { Detsky AS, Abrams HB, McLaughlin JR, Drucker DJ, Sasson Z, } \\
\text { Johnston N, et. al. Predicting cardiac complications in patients } \\
\text { undergoing noncardiac surgery. J Gen Int Med. 1986;1(4):211- } \\
9^{(11)} \text {. }\end{array}$ \\
\hline & $\begin{array}{l}\text { E4 - Estratificação de } \\
\text { risco da American } \\
\text { College of Cardiology } \\
\text { e pela American Heart } \\
\text { Association }\end{array}$ & $\begin{array}{l}\text { Antman E, Bassand JP, Klein W, Ohman M, Sendon JLL, Rydén } \\
\text { L,et al. Myocardial infarction redefined - a consensus document } \\
\text { of The Joint European Society of Cardiology/American College } \\
\text { of Cardiology Committee for the redefinition of myocardial } \\
\text { infarction. J Am CollCardiol. 2009;36(3):959-69 } 9^{(12)} \text {. }\end{array}$ \\
\hline $\begin{array}{l}\text { Risco Cirúrgico de } \\
\text { Trombose Venosa } \\
\text { Profunda (TVP) e } \\
\text { Tromboembo-lismo } \\
\text { Pulmonar (TP) } \\
\end{array}$ & $\begin{array}{l}\text { E5 - Programa de } \\
\text { Avaliação Perioperatória } \\
\text { do idoso (PROAPI) }\end{array}$ & $\begin{array}{l}\text { Sitta MC, Machado NA,Apolinario D, Leme DEG. Avaliação } \\
\text { perioperatória do idoso. Geriatria \& Gerontologia. 2008;2(2):86- } \\
94^{(13)} \text {. }\end{array}$ \\
\hline $\begin{array}{l}\text { Risco Cirúrgico } \\
\text { Pulmonar }\end{array}$ & $\begin{array}{l}\text { E6 - Índice Multifatorial } \\
\text { de risco para Insuficiência } \\
\text { Respiratória no Pós- } \\
\text { Operatório }\end{array}$ & $\begin{array}{l}\text { Faresin SM, Barros JA, Beppu OS, Peres CA, Atallah AN. } \\
\text { Aplicabilidade da escala de Torrington e Henderson. } \\
\text { RevAssocMed Bras. } 2000 ; 46(2): 159-65^{(14)} \text {. }\end{array}$ \\
\hline $\begin{array}{l}\text { Risco Cirúrgico de } \\
\text { Prejuízo Cognitivo }\end{array}$ & $\begin{array}{l}\text { E7 - MEEM (Mini Exame } \\
\text { do Estado Mental) }\end{array}$ & $\begin{array}{l}\text { Folstein MF, Folstein SE, McHugh PR. Mini-mental state: a } \\
\text { practical method for grading the cognitive state of patients for } \\
\text { the clinician. J Psychiatric Res. 1975;(12):189-98 }{ }^{(15)} \text {. }\end{array}$ \\
\hline \multirow[t]{4}{*}{$\begin{array}{l}\text { Risco de Úlcera Por } \\
\text { Pressão (UP) }\end{array}$} & E8 - Escala de Braden & $\begin{array}{l}\text { Bergstrom N, Braden BJ, Laguzza A, Holman V. The Braden Scale } \\
\text { for predicting pressure sore risk. Nurs Res. 1987;36(4):205-10 }{ }^{(16)} \text {. }\end{array}$ \\
\hline & E9 - Escala de Norton & $\begin{array}{l}\text { Kwong E, Pang S, Wong T, Ho J, Shao-Ling X, Li-jun T. Predicting } \\
\text { pressure ulcer risk with the modified Braden, Braden, and Norton } \\
\text { scales in acute care hospitals in Mainland China. ApplNurs Res. } \\
2005 ; 18(2): 122-8^{(17)} \text {. }\end{array}$ \\
\hline & E10 - Escala de Gosnell & $\begin{array}{l}\text { Meesterberends E, Haffens R, Lohrmann C, de Wit R. Pressure } \\
\text { ulcer guideline development and dissemination in Europe. J } \\
\text { ClinNurs. 2010;19(11-12):1495-1503 }{ }^{(18)} \text {. }\end{array}$ \\
\hline & E11 - Escala de Waterlow & $\begin{array}{l}\text { Sayar S, Turgut S, Dogan H, Ekici A, Yurtsever S, Demirkan F, et } \\
\text { al. Incidence of pressure ulcers in intensive care unit patients at } \\
\text { risk according to the Waterlow scale and factors influencing the } \\
\text { development of pressure ulcers. J ClinNurs. 2007;18(5):765-74 }{ }^{(19)} \text {. }\end{array}$ \\
\hline \multirow[t]{4}{*}{ Risco de Quedas } & E12 - Escala de Morse & $\begin{array}{l}\text { Morse JM, Morse RM, Tylko SJ. Development of a scale to identify } \\
\text { the fall-prone patient. Adaptação cultural e linguística, } 2010 . \\
\text { Centro de Estudos e Investigação em Saúde da Universidade } \\
\text { de Coimbra (CEISUC). Canadian Journalon Aging. 1989;(8):366- } \\
77^{(20)} \text {. }\end{array}$ \\
\hline & E13 - Escala de Schmid & $\begin{array}{l}\text { Schmid NA. Reducing patients falls: A research-based } \\
\text { comprehensive fall prevention program. Military Medicine. } \\
\text { 1990;155(2):202-7 }\end{array}$ \\
\hline & E14 - Escala de Downton & $\begin{array}{l}\text { Summary of the updated American Geriatrics Society/British } \\
\text { Geriatrics Society clinical practice guideline for prevention of } \\
\text { falls in older persons. Journal of the American Geriatrics Society. } \\
2011 ; 59(1): 148-57^{(22)} \text {. }\end{array}$ \\
\hline & $\begin{array}{l}\text { E15 - Escala de Hendrich } \\
\text { II }\end{array}$ & $\begin{array}{l}\text { Hendrich AL, BenderSP,Nyhuis A. Validation of the Hendrich } \\
\text { II Fall Risk Model: a large concurrent case/control study of } \\
\text { hospitalized patients. AppliedNursingResearch. 2003;16(1):9- } \\
21^{(23)} \text {. }\end{array}$ \\
\hline
\end{tabular}




\begin{tabular}{|c|c|c|}
\hline \multirow[t]{4}{*}{ Risco de Dor } & $\begin{array}{l}\text { E16 - Escala de Estimativa } \\
\text { Numérica (Numeric } \\
\text { Rating Scale - NRS) }\end{array}$ & $\begin{array}{l}\text { Sousa FF, Silva JA. A métrica da dor (dormetria): problemas } \\
\text { teóricos e metodológicos. Revista DOR. 2005;6(1):469-513 }{ }^{(24)} \text {. }\end{array}$ \\
\hline & $\begin{array}{l}\text { E17 - Escala Analógica } \\
\text { Visual (Visual Analogue } \\
\text { Scale) }\end{array}$ & $\begin{array}{l}\text { Collins SL, Moore RA, Mcquay HJ. The visual analogue pain } \\
\text { intensity scale What is moderate pain in millimeters? Pain. } \\
\text { 1997; }(72): 95-7^{(25)} \text {. }\end{array}$ \\
\hline & $\begin{array}{l}\text { E18 - Escalas de } \\
\text { Categorias Verbais ou } \\
\text { Visuais (Verbal - Visual } \\
\text { Rating Scale) }\end{array}$ & $\begin{array}{l}\text { Pimenta CI. Fundamentos teóricos da dor e de sua avaliação. } \\
\text { In: Carvalho MMJ. (Org). Dor: um estudo multidisciplinar. São } \\
\text { Paulo: Summus; 1999. p. 31-46 }{ }^{(26)} \text {. }\end{array}$ \\
\hline & $\begin{array}{l}\text { E19 - Escalas (Categoria } \\
\text { - Razão) de Borg para } \\
\text { mensuração da dor (Borg } \\
\text { CR Scales) } \\
\text { E20 - Escala } \\
\text { Multidimensional da dor } \\
\text { (McGill) }\end{array}$ & $\begin{array}{l}\text { Melzack R, Katz J. The McGill Pain Questionnaire: Appraisals } \\
\text { and current status. In: Turk DG. e Melzack R. Handbook of Pain } \\
\text { Assessment. New York: Guilford; 1992. p.152- } 65^{(27)} \text {. }\end{array}$ \\
\hline Risco Anestésico & $\begin{array}{l}\text { E21 - Escala de Aldret e } \\
\text { Kroulik }\end{array}$ & $\begin{array}{l}\text { Americam Society of Anesthesiologist (ASA). Physical status } \\
\text { classification system Washington; } 2011^{(28)} \text {. }\end{array}$ \\
\hline $\begin{array}{l}\text { Risco de Bloqueio } \\
\text { Motor }\end{array}$ & E22 - Escala de Bromage & $\begin{array}{l}\text { PasinS,Schnath F. Cuidados de enfermagem na analgesia por } \\
\text { cateter peridural. Rev HCPA. 2007;27(2):69-73 }\end{array}$ \\
\hline
\end{tabular}

de aplicar concomitantemente várias das escalas. A Figura 1 ilustra esquema dos instrumentos para identificação dos riscos cirúrgicos mais comuns.

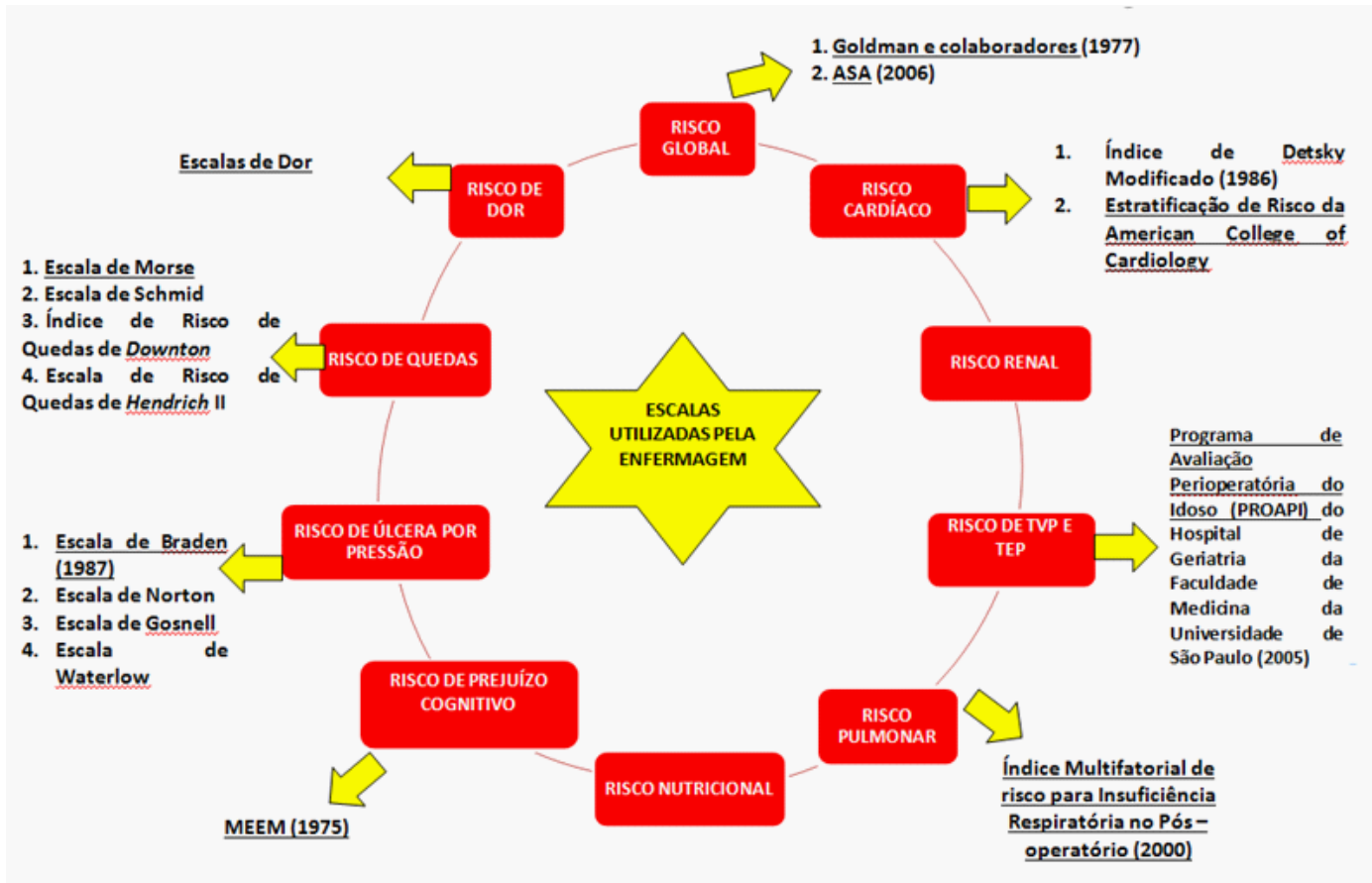

Figura 1 - Risco cirúrgico e escalas de avaliação. Florianópolis, SC, Brasil, 2016

\section{DISCUSSÃO}

Em relação à avaliação da potencialidade de riscos inerentes à cirurgia em idosos, atenção particular deve ser conferida às comorbidades, fatores de risco cardíaco para tromboembolismo e história medicamentosa, além de outros aspectos que envolvam modificações morfológicas e funcionais do organismo senescente ${ }^{(1)}$.

Aspecto primordial a ser avaliado é o risco cardíaco. Ao ser submetido a estresse cirúrgico, 
idosos podem evidenciar reserva cardiovascular deficitária. Ademais, a hipertensão arterial, com alta prevalência entre idosos, é problema comum nesses pacientes em condição cirúrgica ${ }^{(1)}$. Estudo que avaliou o risco cardiovascular de portadores de hipertensão arterial evidenciou que o maior percentual de indivíduos com risco cardiovascular situou-se acima de 70 anos e o médio risco acima de 60 anos ${ }^{(30)}$. Foram identificadas duas escalas (E3, E4) com o propósito de avaliação do quesito cardiovascular, que podem ser aplicados em idosos.

A escala de avaliação pulmonar (E6) também se configura como importante ferramenta que pode ser utilizada pelo enfermeiro em seu arsenal de avaliação do idoso, uma vez que este é mais propenso às complicações respiratórias, dadas as alterações fisiológicas decorrentes do envelhecimento ${ }^{(1)}$.

A escala que avalia o risco de TVP e TEP (E5) deve ser amplamente utilizada tendo em vista que a incidência de TVP aumenta de 1.8/1000 indivíduos com idade entre 65 a 69 anos para 3.1/1000 indivíduos entre 85 e 90 anos, e o TEP é a complicação tromboembólica mais comum em pós-operatórios, frequentemente assintomática ${ }^{(1,31)}$.

As escalas para avaliar risco de UP (E8, E9, E10, E11) são essenciais, pois a pele do idoso fica mais sensível e predisposta a sofrer alterações significativas, especialmente em decorrência do tempo do procedimento cirúrgico e do posicionamento cirúrgico. Nessa perspectiva, as úlceras por pressão são causadas pela interrupção de suprimento sanguíneo, geralmente, provocadas por pressão, cisalhamento ou fricção, ou mesmo por uma combinação desses fatores ${ }^{(32)}$.

Sobre os riscos de quedas, trata-se de intervenção de relevância para o enfermeiro implementar no cuidado ao idoso cirúrgico, uma vez que este evento pode estar condicionado a fatores relacionados ao paciente (intrínsecos), ao ambiente hospitalar e ao processo de trabalho dos profissionais (extrínsecos) (33).

As demais escalas que avaliarão dor, risco de bloqueio motor e anestésico devem ser implementadas, pois o idoso possui maior risco de delirium e alteração do nível de consciência, principalmente na faixa etária de 60 a 69 anos $^{(34)}$.

A avaliação do risco cirúrgico configura-se como condição necessária para garantir a segurança do paciente idoso. Para tanto, as ações no cuidado ao mesmo no período perioperatório devem estar pautadas nas recomendações do Programa Nacional de Segurança do Paciente (PNSP). Essa abordagem tenta repensar os processos de atendimento, no sentido de antecipar a ocorrência de erros antes que eles causem dano ao paciente ${ }^{(35)}$.

Idosos frequentemente são mais vulneráveis quando hospitalizados, especialmente em condição cirúrgica, que por si só, engloba situação de risco. Vários estudos denotam que esta população é mais propensa aos eventos adversos evitáveis e a danos causados pela assistência hospitalar ${ }^{(36)}$. As complicações da cirurgia do paciente idoso serão focalizadas em decorrência do envelhecimento, ou seja, menor adaptabilidade às mudanças do meio ambiente e da sua própria homeostase em consequência da redução global de sua reserva orgânica ${ }^{(37)}$. Portanto, é essencial que seja proporcionado a eles cuidado técnico de qualidade e que lhes traga segurança ${ }^{(38)}$.

\section{CONSIDERAÇÕES FINAIS}

A utilização de escalas de avaliação de risco do idoso em condição perioperatória possibilita fundamentar os princípios da segurança do paciente, pois propicia ao enfermeiro realizar avaliação global, identificando fatores de risco e, por conseguinte, possibilitando a prevenção de complicações cirúrgicas. Contudo, identificam-se lacunas de conhecimento que apontam a incipiente realização de pesquisas no âmbito da Enfermagem.

\section{REFERÊNCIAS}

1. Vendites S, Almada-Filho CM, Minosse JG. Aspectos gerais da avaliação pré-operatória do paciente idoso cirúrgico. ABCD, arq. bras. cir. dig. 2010; 23(3): 173-82. 
2. Schulz RS, Santana RF, Faleiro TB, de Carvalho MR, Gonçalves RCS, Alves LAF. Necessidade de movimentarse e manter uma boa postura no idoso cirúrgico: quase-experimento. Rev. pesqui. cuid. fundam. (Online). 2014; 6(1): 398-407.

3. Cauduro FLF, Sarquis LM, Sarquis LMM, Cruz EDA. Cultura de segurança entre profissionais de centro cirúrgico. CogitareEnferm. [Internet] 2015; 20(1) [acesso em 10 abr 2016]. Disponível:

http://dx.doi.org/10.5380/ce.v20i1.36645.

4. Vitor AF, Moura LA, Fernandes APNL, Botarelli FR, Araújo JNM, Vitorino ICC. Risco de quedas em pacientes no período pós-operatório. CogitareEnferm. [Internet] 2015; 20(1) [acesso em 10 abr 2016]. Disponível: http://dx.doi.org/10.5380/ce.v20i1.38509.

5. Mendoza IYQ, Peniche ACG. Conhecendo o perfil do idoso cirúrgico. Saúde Colet. 2009; 6(30): $104-8$.

6. Fernandes EO, Guerra EE, Pitrez FAB, Fernandes FM, Rosito GBA, Gonzàles HE, et al. Avaliação pré-operatória e cuidados em cirurgia eletiva: recomendações baseadas em evidências. Rev. AMRIGS. 2010; 54(2): 240 -58.

7. Pfützenreuter AH, Alvim ATB. O direito à cidade: as diretrizes políticas mundiais para o envelhecimento. Revista Nacional de Gerenciamento das Cidades. 2015; 3(14): 112-27.

8. Severino AJ. Metodologia do trabalho científico. 23ª ed. São Paulo: Cortez; 2007.

9. Goldman L, Caldera DL, Nussbaum SR, Southwick FS, Krogstad D, Murray B, et al. Multifactorial index of cardiac risk in noncardiac surgical procedures. N Engl J Med. 1977; 297(16): 845-50.

10. Owens WD. American Society of Anesthesiologists. Physical Status Classification System Is Not a Risk Classification System. Anesthesiology. 2001; 94(2): 378.

11. Detsky AS, Abrams HB, McLaughlin JR, Drucker DJ, Sasson Z, Johnston N, et al. Predicting cardiac complications in patients undergoing noncardiac surgery. J Gen Intern Med. 1986; 1(4): 211-9.

12. Antman E, Bassand JP, Klein W, Ohman M, Sendon JLL, Rydén L, Simoons M, Tendera M. Myocardial infarction redefined - a consensus document of The Joint European Society of Cardiology/American College of Cardiology Committee for the redefinition of myocardial infarction. J Am CollCardiol. 2009; 36(3): 959-69.

13. Sitta MC, Machado AN, Apolinario D, Leme LEG.Avaliação perioperatória do idoso. Geriatria \& Gerontologia. 2008; 2(2): 86-94.

14. Faresin SM, de Barros JA, Beppu OS, Peres CA, Atallah AN. Aplicabilidade da escala de Torrington e Henderson. RevAssocMed Bras. 2000; 46(2): 159-65.

15. Folstein MF, Folstein SE, McHugh PR. Mini-mental state: a practical method for grading the cognitive state of patients for the clinician. J Psychiatric Res. 1975; 12(3): 189-98.

16. Bergstrom N, Braden BJ, Laguzza A, Holman V. The Braden Scale for predicting pressure sore risk. Nurs Res. 1987; 36(4): 205-10.

17. Kwong E, Pang S, Wong T, Ho J, Shao-Ling X, Li-jun T. Predicting pressure ulcer risk with the modified Braden, Braden, and Norton scales in acute care hospitals in Mainland China. ApplNurs Res. 2005; 18(2): $122-8$.

18. Meesterberends E, Haffens R, Lohrmann C, de Wit R. Pressure ulcer guideline development and dissemination in Europe. J ClinNurs. 2010; 19(11-12): 1405-503.

19. Sayar S, Turgut S, Dogan H, Ekici A, Yurtsever S, Demirkan F, et al. Incidence of pressure ulcers in intensive care unit patients at risk according to the Waterlow scale and factors influencing the development of pressure ulcers. J ClinNurs. 2007; 18(5): 765-74.

20. Morse JM, Morse RM, Tylko SJ. Development of a scale to identify the fall-prone patient. Adaptação cultural e linguística, 2010. Centro de Estudos e Investigação em Saúde da Universidade de Coimbra (CEISUC). CanadianJournalonAging. 1989; (8): 366-77.

21. Schmid NA.Reducing patients falls: A research-based comprehensive fall prevention program. Mil Med. 1990; 155(2): 202-7. 
22. Panel on Prevention of Falls in Older Persons, American Geriatrics Society and British Geriatrics Society. Summary of the updated American Geriatrics Society/British Geriatrics Society clinical practice guideline for prevention of falls in older persons. J Am Geriatr Soc. 2011; 59(1): 148-57.

23. Hendrich AL, Bender PS, Nyhuis A. Validation of the Hendrich II Fall Risk Model: a large concurrent case/ control study of hospitalized patients. App Nurs Res. 2003; 16(1): 9-21.

24. Sousa FF, Silva JA. A métrica da dor (dormetria): problemas teóricos e metodológicos. Revista DOR. 2005; 6(1): 469-513.

25. Collins SL, Moore RA, McQuay HJ. The visual analogue pain intensity scale What ismoderate pain in millimeters? Pain. 1997; 72(1-2): 95-7.

26. Pimenta CI. Fundamentos teóricos da dor e de sua avaliação. In: Carvalho MMJ. (Org). Dor: um estudo multidisciplinar. São Paulo: Summus; 1999. p. 31-46.

27. Melzack R, Katz J. The McGill Pain Questionnaire: Appraisals and current status. In: Turk DG, Melzack R. Handbook of Pain Assessment. New York: Guilford; 1992. p.152-65.

28. Americam Society of Anesthesiologist (ASA). Physical status classification system Washington; 2011.

29. PasinS, Schnath F.Cuidados de enfermagem na analgesia por cateter peridural.Rev HCPA. 2007; 27(2): 69-73.

30. Cesarino EJ, Vituzzo ALG, Sampaio JMC, Ferreira DAS, Pires HAF, de Souza L. Assessment of cardiovascular risk of patients with arterial hypertension of a public health unit. Einstein. 2012; 10(1): 33-8.

31. Engelhorn CA, Nardelli J, Iwamura APD, Salgado LSA, Hartmann MO, Witt NC. Drug prophylaxis of deep vein thrombosis in patients submitted to trauma surgery in a university hospital. J.vasc. bras. 2012; 11(2): 97-101.

32. Coelho ADA, Lopes MVO, de Melo RP, de Castro ME. O idoso e a úlcera por pressão em serviço de atendimento domiciliar. Rev Rene. 2012; 13(3): 639-49.

33. Severo IM, Almeida MA, Kuchenbecker R, Vieira DFVB, Weschenfelder ME, Pinto LRC, et al. Fatores de risco para quedas em pacientes adultos hospitalizados: revisão integrativa. Rev. esc. enferm. USP. [Internet] 2014; 48(3) [acesso em 13 abr 2016]. Disponível: http://dx.doi.org/10.1590/S0080-623420140000300021.

34. Nascimento PDFS, Bredes AC, De Mattia AL. Complicações em idosos em Sala de Recuperação Pós-Anestésica (SRPA).Rev. SOBECC. 2015; 20(2): 64-72.

35. Acauan LV, Rodrigues MCS. Safety of the elderly patient in cardiac computerized tomography: a descriptive study. Online braz j nurs. [Internet] 2013; 12(Suppl) [acessoem 10 jan 2016]. Disponível:

http://www.objnursing.uff.br/index.php/nursing/article/view/4381/html.

36. Mendes W, Pavão ALB, Martins M, Moura MLO, Travassos C. The feature of preventable adverse events in hospitals in the State of Rio de Janeiro. Rev. Assoc. Med. Bras.[Internet] 2013; 59(5) [acesso em 10 jan 2015]. Disponível: http://dx.doi.org/10.1016/j.ramb.2013.03.002.

37. Gomes M, Ramacciotti E. Profilaxia do tromboembolismo venoso em cirurgia geral. Rev. Col. Bras. Cir. [Internet] 2002; 2(1) [acesso em 10 jan 2015]. Disponível:

https://cbc.org.br/wp-content/uploads/2013/05/Ano2-I.Tromboembolismo-venoso.pdf.

38. Prochet TC, da Silva MJP. Percepção do idoso dos comportamentos afetivos expressos pela equipe de enfermagem. Esc Anna Nery. [Internet]2011; 15(4) [acesso em 15 dez 2015]. Disponível:

http://dx.doi.org/10.1590/S1414-81452011000400018. 